\title{
Beneficial effects of treatment with transglutaminase inhibitor cystamine on the severity of inflammation in a rat model of inflammatory bowel disease
}

\author{
Luca Elli ${ }^{1}$, Michele M Ciulla ${ }^{2}$, Giuseppe Busca ${ }^{2}$, Leda Roncoroni ${ }^{3}$, Claudio Maioli ${ }^{4}$, Stefano Ferrero ${ }^{5}$, \\ Maria Teresa Bardella ${ }^{1,3}$, Antonella Bonura ${ }^{3}$, Roberta Paliotti ${ }^{2}$, Claudia Terrani ${ }^{6}$ and Paola Braidotti ${ }^{5}$
}

Inflammatory bowel disease (IBD) represents a socially and clinically relevant disorder, characterized by intestinal chronic inflammation. Cystamine (CysN) is a multipotent molecule with healthy effects and, moreover, it is an inhibitor of transglutaminases (TGs), including the TG type 2 (TG2), an enzyme with pleiotropic functions, involved in different pathways of inflammation and central in the pathogenesis of some human disorders as the IBD. Our aim was to evaluate the effect of Cys N in an IBD rat model. A total of 30 rats were divided into 4 groups: controls without treatment (CTR; $n=7$ ); receiving the 2,4,6-trinitrobenzene sulfonic acid enema (TNBS group; $n=8$ ); treated with TNBS enema plus oral CysN (TNBS-CysN group; $n=8$ ); treated with CysN (CysN group; $n=7$ ). After killing, bowel inflammation was evaluated applying specific scores. TG activity, TG2 and isopeptide bond immunohistochemical expression, and tumor necrosis factor- $\alpha$ (TNF- $\alpha$ ) were evaluated in the colonic tissue, such as interleukin-6 (IL-6) serological levels (ELISA). TG2 was also evaluated on the luminal side of the colon by immunoautoradiography. Colonic samples from IBD patients were compared with animal results. TNBS-CysN group developed a less severe colitis compared with the TNBS group (macroscopic score $0.43 \pm 0.78$ vs $3.28 \pm 0.95$, microscopic score $6.62 \pm 12.01$ vs $19.25 \pm 6.04, P<0.05$, respectively) associated with a decrease of TG activity, TG2 and isopeptide bond immunohistochemical expression, TNF- $\alpha$ and IL- 6 levels. No statistically significant differences were found between Cys $\mathrm{N}$ and CTR groups. The colonic immunolocalization of TG2 was comparable in humans affected by IBD and TNBS-administered animals. This is the first demonstration that treatment with a Cys $\mathrm{N}$ has an anti-inflammatory effect, reducing severity of colitis in a rat model. Cys $\mathrm{N}$ could be tested as a possible treatment or co-treatment in IBD therapeutic trials.

Laboratory Investigation (2011) 91, 452-461; doi:10.1038/labinvest.2010.186; published online 1 November 2010

KEYWORDS: colitis; cystamine; inflammatory bowel disease; transglutaminase type 2

Inflammatory bowel disease (IBD) is an idiopathic chronic intestinal disorder, mainly divided into two major forms, ulcerative colitis (UC) and Crohn's disease (CD). In the Western countries, IBD represents a socially relevant disorder with an estimated incidence of $\sim 100 /$ million/year, increasing in the last decades in both males and females. Clinical presentation is heterogeneous, ranging from paucisymptomatic subclinical patterns to severe pictures complicated by intestinal perforation, visceral stenosis and/or enteric fistulae. Macroscopically, the intestinal lesions are characterized by hyperemia, loss of the submucosal vascular pattern, friability, cobblestone appearance, aphths and ulcers. Histological damage is dominated by an active inflammation, limited to the mucosa and submucosa layers of colon and rectum in UC, and throughout the intestinal wall of the whole gastrointestinal tract in CD. ${ }^{1,2}$

A multifactorial ethiopathogenesis is suggested by different studies demonstrating that genetic and environmental factors cooperate in triggering the immunological system that finally induces the characteristic gut mucosal damage and the

\footnotetext{
${ }^{1}$ Centro per la Prevenzione e Diagnosi della Malattia Celiaca, Fondazione IRCCS Cà-Granda Ospedale Maggiore Policlinico Milano, Milano, Italy; ${ }^{2}$ Dipartimento Toracopolmonare e Cardiocircolatorio, Università degli Studi di Milano, Milano, Italy; ${ }^{3}$ Dipartimento di Scienze Mediche, Università degli Studi di Milano, Milano, Italy; ${ }^{4}$ Dipartimento di Scienze e Tecnologie Biomediche, Sezione di Scienze Radiologiche, Università degli Studi di Milano, Milano, Italy; ${ }^{5}$ Dipartimento di Medicina, Chirurgia e Odontoiatria, Ospedale San Paolo e Fondazione IRCCS Cà-Granda Ospedale Maggiore Policlinico Milano, Università degli Studi di Milano, Milano, Italy and ${ }^{6}$ Gastroenterologia 2, Fondazione IRCCS Cà-Granda Ospedale Maggiore Policlinico Milano, Milano, Italy

Correspondence: Dr L Elli, MD, PhD, Centro per la Prevenzione e Diagnosi della Malattia Celiaca, Fondazione IRCCS Cà-Granda Ospedale Maggiore Policlinico, Via F. Sforza 35, Milano, 20122, Italy.
}

E-mail: lucelli@yahoo.com

Received 22 December 2009; revised 30 September 2010; accepted 30 September 2010 
systemic clinical manifestations. ${ }^{3}$ Although largely unknown, the IBD pathomechanisms are linked to an impairment of the intestinal immune system driven from the presence of the normal luminal flora. By now, the unique widely accepted therapy for IBD is based on the use of anti-inflammatory and immunosuppressive drugs that are sometimes ineffective (nonresponder patients) or present important side effects. For these reasons, new therapies are widely requested by patients and clinicians. ${ }^{4-6}$ Among the possible pharmaceutical molecules, cystamine $(\mathrm{Cys} N)$ appears to be a candidate because of its effects on apoptosis (inhibition of caspase 3), redox balance (increase of intracellular glutathione) and, above all, on transglutaminases (TGs); ${ }^{7,8}$ in fact, CysN is a well-known and available inhibitor of TG enzymatic family, including the TG type 2 (TG2), a calcium-dependent enzyme widely expressed in the gut mucosa with pleiotropic functions. TG2 transamidates (catalyzing stable [( $\gamma$-glutamyl)lysine isopeptide bonds) and deamidates, displays G-protein, protein disulfide isomerase and protein kinase activities, and forms tight complexes with fibronectin, integrins and other proteins of the extracellular matrix. ${ }^{9,10}$ Thus, it is actually considered pivotal in degenerative ${ }^{11,12}$ and autoimmune disorders, ${ }^{13}$ infection, ${ }^{14,15}$ fibrosis ${ }^{16}$ and also in IBD, where it is increased in the lamina propria of the damaged mucosa and is targeted by autoantibodies present in a relevant percentage of patients. ${ }^{17-19}$

To test new potentially effective drugs in IBD, a widely used animal model is the colitis induced by the 2,4,6-trinitrobenzene sulfonic acid (TNBS) enema that causes a chronic mucosal inflammation sharing several immunologic similarities with $\mathrm{IBD}^{20-23}$

The aim of this study was to investigate the effects of CysN treatment on the rat TNBS model of IBD.

\section{MATERIALS AND METHODS Animals}

A total of 30 male adult Sprague-Dawley rats, weighting 220-250 g, were purchased from Charles River Laboratories (Calco, Italy) and used for the experiments. Animals were housed and handled in accordance with the National Institute of Health Guide for the Care and Use of Experimental Animals. In particular, they were maintained in a restricted access room with temperature control at $26^{\circ} \mathrm{C}$ and dark-light cycle was $12 / 12 \mathrm{~h}$. Rats were housed in pairs in polycarbonate cages and had free access to water and standard chow. To ensure the permanent identification, a microchip device (Avid Microchip MUSICC, Barcelona, Spain) was implanted subcutaneously in each rat.

The study was approved by the ethical committee of the 'Fondazione IRCCS Cà-Granda Ospedale Maggiore' of Milano.

\section{Induction of Colitis}

Colitis was induced by intracolonic administration of TNBS (Sigma Aldrich, Milano, Italy) as described by Morris et al. ${ }^{24}$
After an overnight fast, a single dose of $40 \mathrm{mg}$ TNBS in 50\% ethanol (total volume $0.5 \mathrm{ml}$ ) was directly instilled into the colon using a polyethilene catheter (diameter $0.8 \mathrm{~mm}$ ) inserted intrarectally for $8 \mathrm{~cm}$. To ensure a sufficient contact time of the active agent, after catheter removal, the animals were kept in headlong vertical position for $2 \mathrm{~min}$.

\section{Study Design}

Rats were randomly divided into four different groups: the control group (CTR; $n=7$ ) with the instillation of vehicle only; the TNBS group $(n=8)$ treated with TNBS enema; the TNBS-CysN group $(n=8)$ treated with TNBS enema plus oral CysN administration; and the CysN group $(n=7)$ treated with CysN. CysN powder (Sigma Aldrich) was dissolved in the drinking water $(1 \mathrm{~g} / \mathrm{l})$ of the CysN and TNBS-CysN groups, starting 14 days before the induction of colitis (day 0). On day 0, TNBS and TNBS-CysN groups received acid enema. On day 8 , the rats were killed by decapitation after sedation. Colon was extracted from the abdomen, longitudinally cut and macroscopically evaluated; tissue samples were taken from the left, transverse and right colon and collected into $2.5 \mathrm{ml}$ tubes (Eppendorf, Milano, Italy), frozen by liquid nitrogen immersion and stored at $-80^{\circ} \mathrm{C}$ till the analysis. The remnant was formalin fixed $(10 \%)$. Following the same procedures, two supplementary groups of seven rats were killed after $24 \mathrm{~h}$ from a TNBSinduced colitis with or without CysN treatment (TNBS-24 h group and TNBS-CysN-24h group). These two groups were added to check a possible biochemical interference between TNBS and CysN leading to a reduced acid effect on colonic tissue.

The study design scheme is shown in Figure 1.

\section{Macroscopic Scoring of Colitis}

After the excision of colon and its longitudinal opening, it was pinned on a board and macroscopically evaluated by two

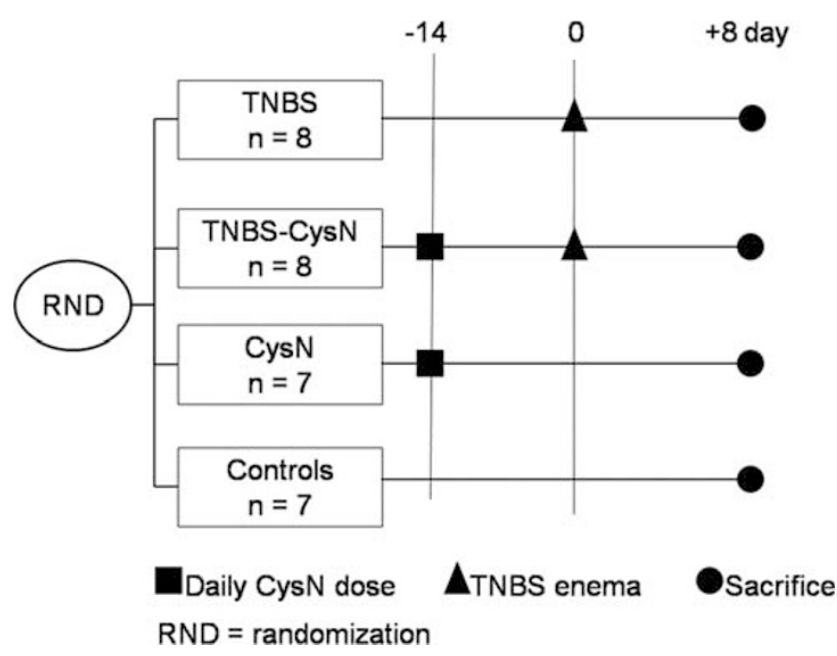

Figure 1 Study design scheme. 
expert pathologists (SF, BP). The inflammation was scored on a scale of $0-4$, as previously described by Morris et al, ${ }^{24}$ as follows: 0 , no signs of inflammation; 1, erythema; 2, erythema, edema and small erosions; 3 , two or more bleeding ulcers, inflammation and adhesions; and 4, severe ulcerations and/or stenosis with dilatation of the proximal intestine.

\section{Microscopic Scoring of Colitis}

After formalin fixation, each sample was rolled up in a spiral from the cranial (central part) to the distal anal extremity (external part) locked in tissue cassettes routinely processed and paraffin embedded. Hematoxylin-eosin (HE) colored sections, in which all colonic wall thickness was visible from mucosa to serosa, were blindly observed by the two pathologists for histological analysis. In each rat, tissue damage score was assessed in four colonic tracts (ascending, transverse, descending and sigma-rectum) applying a modified method of Gaudio et $a^{25}$ (Table 1); the final score was reported as sum of the single scores (maximum damage $=36$ ).

\section{Immunohistochemistry}

Immunoreactivity of paraffin-embedded sample sections was tested by incubation in an automated slide stainer (Biogenex, San Ramon, CA, USA) with 1:5000 mouse monoclonal antiTG2 antibody clone TG 100 (Thermo Fisher Scientific, Fremont, CA, USA), with 1:200 polyclonal goat anti-rat TNF- $\alpha$ antibody (Santa Cruz Biotechnology, Heidelberg, Germany) or with 1:250 mouse monoclonal anti-isopeptide bond (Abcam, Milano, Italy) antibodies. Previous antigen retrieval was obtained by $30 \mathrm{~min}$ in $95^{\circ} \mathrm{C}$ citrate buffer immersion and reaction products were visualized by Envision (Dako Cytomation, Glostrup, Denmark). Diaminobenzidine (DAB) brown staining has been used to reveal antibodies reaction. Positive and negative controls were added. ${ }^{26}$

\section{Transglutaminase Type $\mathbf{2}$ Immunoautoradiography} Immunoautoradiography (IAR) was performed following a modified method described by Herman and Morrison. ${ }^{27}$ Briefly, frozen colonic fragments were warmed at room temperature and incubated with rabbit serum 1:30 for $30 \mathrm{~min}$; they were washed three times with PBS and successively incubated with 1:1000 anti-TG2 antibody (clone TG 100; Thermo Fisher Scientific) overnight at $4{ }^{\circ} \mathrm{C}$. After PBS washing, they were incubated for $1 \mathrm{~h}$ with 1:200 anti-mouse IgG ${ }^{125}$ I radiolabeled antibody (PerkinElmer, Boston, MA, USA), washed three times with PBS, dried and exposed to Kodak Bio Max MS-1 Autoradiography Film for 3 days in appropriate cassettes. Controls included substitution of primary or of secondary antibodies with buffer solution.

\section{Colonic Transglutaminase Activity, TNF- $\alpha$ Mucosal Levels and Serological IL-6}

Tissue samples were weighted (Gibertini E42S, Milano, Italy) and $500 \mathrm{mg}$ homogenized (Ultra Turrax T8 IKA, Staufen, Germany) at $4{ }^{\circ} \mathrm{C}$ in $1 \mathrm{ml}$ of distilled water added with $20 \mu \mathrm{l}$ of protease inhibitor cocktail without EDTA (Pierce, USA). TG2 activity was evaluated by a colorimetric technique (Covalab, Lyon, France) following the manufacturer's instructions. Briefly, $50 \mu \mathrm{l}$ of the obtained homogenized was dispensed in each well of the 96-well microtiter plate with covalently coupled CBZ-Gln-Gly and incubated with calcium, dithiothreitol and biotinylated cadaverine; successively, strepavidin-labeled peroxidase was added to the wells and the peroxidase activity revealed using $\mathrm{H}_{2} \mathrm{O}_{2}$ and tetramethyl benzidine. Optical density was measured at $450 \mathrm{~nm}$ with microplate reader (Bouty Diagnostici, Milan, Italy). The results were normalized referring to tissue weight.

TNF- $\alpha$ was evaluated by a colorimetric technique following the manufacturer's instruction (Bender Medsystems, Wien, Austria). Briefly, anti-TNF- $\alpha$ antibody-coated microwells were incubated with a biotin-conjugated anti-TNF- $\alpha$

Table 1 Microscopic inflammatory score of colonic mucosa

\begin{tabular}{|c|c|c|}
\hline Parameter A & Epithelium and glands & $\begin{array}{l}0=\text { normal } \\
1=\text { focal destruction of epithelial surface and/or glands } \\
2=\text { zonal destruction of epithelial surface and/or zonal crypt loss } \\
3=\text { diffuse mucosal ulceration involving submucosa and/or diffuse crypt loss }\end{array}$ \\
\hline Parameter B & Inflammatory cell infiltration & $\begin{array}{l}0=\text { absence of infiltrate } \\
1=\text { subepithelial and in the lamina propria } \\
2=\text { infiltrate reaches the muscolaris mucosae } \\
3=\text { severe and diffuse infiltrate reaching the submucosa and/or involving the muscolaris propria }\end{array}$ \\
\hline Parameter C & Edema & $\begin{array}{l}0=\text { absent } \\
1=\text { focal } \\
2=\text { zonal and/or moderately diffuse } \\
3=\text { extensive and severe }\end{array}$ \\
\hline
\end{tabular}


antibody, streptavidin-HRP complex and a substrate. Optical density was measured at $450 \mathrm{~nm}$ with microplate reader. The results were normalized referring to tissue weight.

Serum collected after centrifugation $(2200 \mathrm{~g}, 10 \mathrm{~min})$ of coagulated blood was stored in sterile $2.5 \mathrm{ml}$ tubes (Eppendorf) at $-80^{\circ} \mathrm{C}$ until analysis. IL-6 was evaluated by a colorimetric technique following the manufacturer's instruction (Bender Medsystems). Briefly, anti-IL-6 antibodycoated microwells were incubated with a biotin-conjugated anti-IL-6 antibody, streptavidin-HRP complex and the substrate. Optical density was measured at $450 \mathrm{~nm}$ with microplate reader.

\section{Colonic Transglutaminase Activity and Immunohistochemistry in IBD Patients and Controls}

To evaluate if the pattern of mucosal TG2 obtained in rats was comparable with that of humans, 28 endoscopic colonic biopsies from 7 UC patients (4 males, median age 42, range 29-50), 28 from 7 CD patients (5 males, median age 39, range 28-45) and 20 from 10 subjects with negative colonoscopy (5 males, median age 41, range 26-47) were investigated for TG2 mucosal expression and activity. In all, 28 biopsies from IBD patients (taken from active disease sites and from endoscopically normal zones) and 10 from nonIBD subjects were formalin fixed and processed for TG2 immunohistochemistry as described for animal samples. The remnant 28 biopsies from IBD (taken from active and endoscopically normal zones) and 10 from non-IBD subjects were homogenized and processed for TG2 activity as for animal protocol.

\section{Image Acquisition and Analysis}

Autoradiography images of distal colon were acquired and stored on a personal computer (Power Mac G4, $867 \mathrm{Mhz}$, 640 MB RAM; Apple, Cupertino, CA, USA) at 32 bits/pixel for the subsequent analysis. Before the analysis, an automated threshold process was performed on the original black and white images to obtain optimal visualization of texture; images were therefore re-coded by using an appropriate color scale. From each stored image a surface plot (xyz) was obtained by using a specific algorithm developed for ImageJ, a freeware image analysis software developed by W Rasband.

\section{Statistical Analysis}

Data were expressed as mean \pm s.d. or median and range. Comparisons of the data were done using one-way ANOVA. All statistical analyses were performed using a statistical computer software (SPSS 13, SPSS, USA). A P-value of 0.05 was considered significant.

\section{RESULTS \\ Effect of Intracolonic TNBS Administration}

At the time of killing, rats of TNBS group weighted $362.3 \pm 36.3 \mathrm{~g}$ (gain from days 0 to $8,24.0 \pm 10.0 \mathrm{~g}$ ), compared with $380 \pm 33.9 \mathrm{~g}$ (gain from days 0 to $8,38.8 \pm 13.3 \mathrm{~g}$ ) of the CTR group (not statistically significant differences). The TNBS group presented a mild-to-severe colitis from the ciecum to the anus; there were no mortal events or complications because of the enema. Colitis was macroscopically characterized by severe bleeding ulcers in the left colon and erythema and edema in the right part of the large intestine (Table 2); microscopically, inflammation was characterized by focal or extensive destruction ranging from epithelial surface to deeper mucosal ulceration with inflammatory infiltration and/or edema (Table 2). In the TNBS-24 h group, a severe inflammation of the colon was noted with bleeding ulcers and presence of blood clots inside the colon (macroscopic score median 3, range 3 to 4, microscopic score 16, 5-18; Figure 2).

CTR group was characterized by the absence of any macroscopic and microscopic signs of colonic damage (Table 2).

An increase in TNF- $\alpha$ and TG2, as demonstrated by immunohistochemistry and ELISA analysis (Figures 3 and 4), was present in the colon of the TNBS group when compared with those in the CTR group. TG2 activity increase in TNBS rats was also confirmed in situ by the increased presence of isopeptide bonds compared with CTR group, as shown by immunohistochemical analysis (Figure 5). TNF- $\alpha$ and TG2 levels were dependent on the part of the colon analyzed, being higher in the left colon, where acid injury was severe, than in the right one, where inflammation was mild. IL-6 serological levels were increased in the TNBS group rats compared with CTR group (Figure 6).

In severe inflamed zones, an increase in TG2 expression was observed at immunohistochemistry in the submucosal compartment and on the luminal surface, as showed by IAR (Figure 7).

Table 2 Macroscopic and microscopic inflammation scores (median and range) of colonic mucosa in the different rat groups

\begin{tabular}{|c|c|c|c|c|c|c|c|}
\hline & TNBS $(n=8)$ & TNBS-CysN $(n=8)$ & CysN $(n=7)$ & CTR $(n=7)$ & TNBS vs TNBS-CysN & TNBS vs CTR & TNBS vs CysN \\
\hline Macroscopic score & $4(2-4)$ & $0(0-2)$ & $0(0-3)$ & $0(0-1)$ & $P<0.0001$ & $P<0.0001$ & $P<0.0001$ \\
\hline
\end{tabular}

TNBS, 2,4,6-trinitrobenzene sulfonic acid; CysN, cystamine; CTR, controls.

Statistically significant differences are reported. 


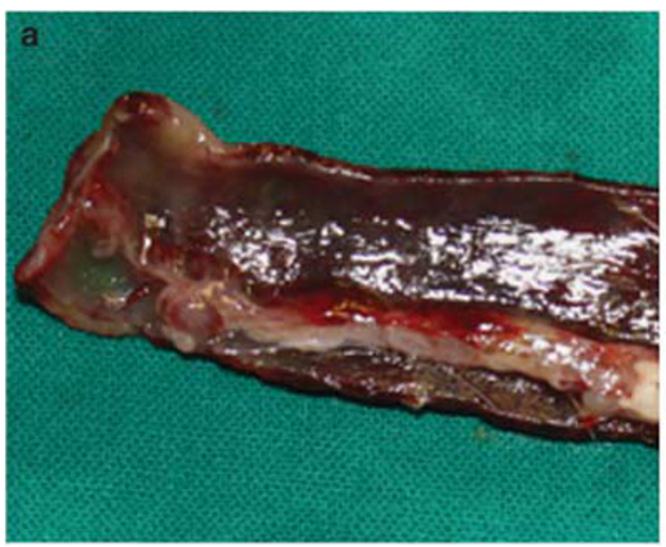

a1

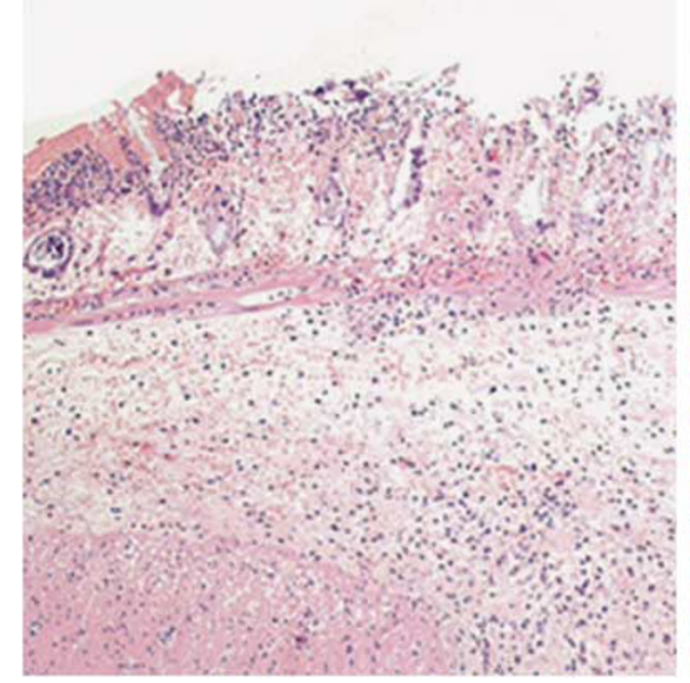

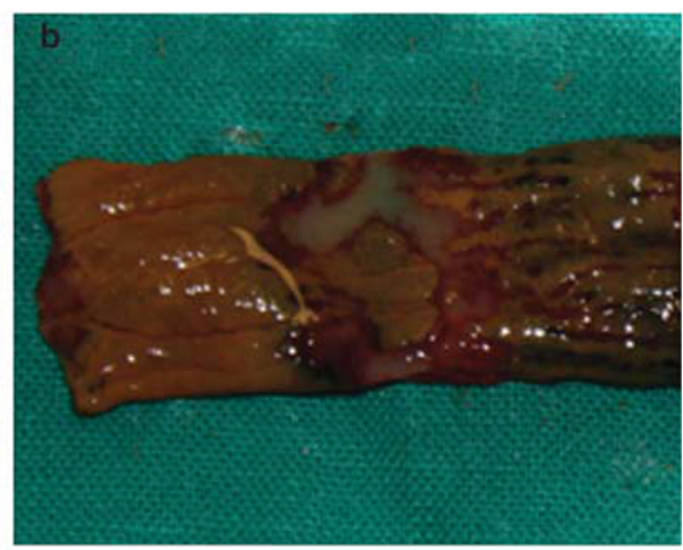

b1

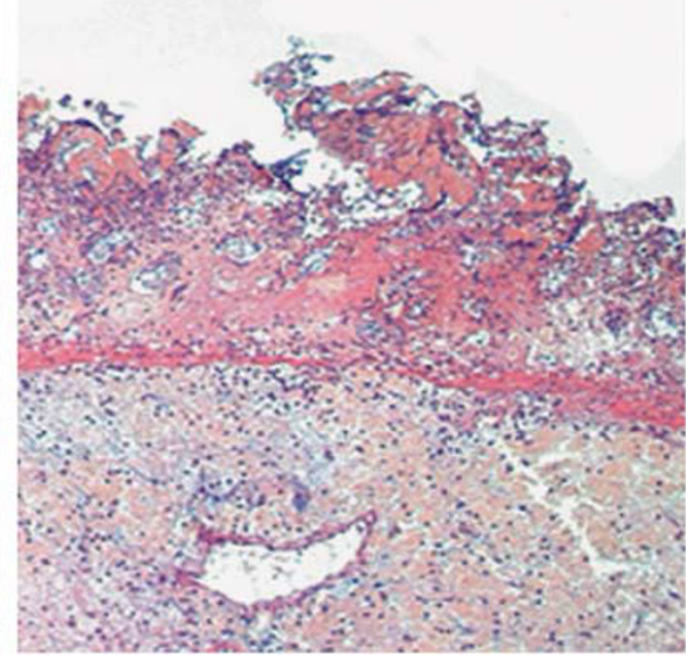

Figure 2 Macroscopic appearance and histology of the colon extracted from the TNBS-24h and TNBS-CysN-24h groups (a, b, a1, b1, respectively).

TNBS-24 h group presented significantly increased mucosal levels of TNF- $\alpha(2.10 \pm 0.21 \mathrm{pg} / \mathrm{mg}$ of tissue; average between rectum, transverse and ascending) and TG activity ( $3.57 \pm 0.53 \mathrm{microU} / \mathrm{mg}$ of tissue; average between rectum, transverse and ascending) and serological levels of IL-6 $(7.12 \pm 0.75 \mathrm{pg} / \mathrm{ml})$ compared with CTR and CysN groups.

In IBD patients, TG2 increase was localized in the mucosal and submucosal layers, and both the epithelial colonocytes and inflammatory cells presented an intracellular TG2 increase. Very low TG2 expression was observed in CTR animal group and in the colonic mucosa of healthy subjects (data not shown). Also, mucosal TG activity was significantly increased in active UC and $\mathrm{CD}$ compared with healthy controls $(1.11 \pm 0.09 \mathrm{microU} / \mathrm{mg}$ of tissue, $1.62 \pm 0.25 \mathrm{microU} / \mathrm{mg}$ of tissue and $0.63 \pm 0.13 \mathrm{microU} / \mathrm{mg}$ of tissue, respectively).

\section{Effect of Cystamine Treatment}

At the time of killing, rats of the TNBS-CysN and CysN groups weighted $381.1 \pm 30 \mathrm{~g}$ (gain from days 0 to 8 , $25.3 \pm 9.8 \mathrm{~g}$ ) and $387.6 \pm 20.3 \mathrm{~g}$ (gain from days 0 to 8 ,
$34.7 \pm 14.7 \mathrm{~g}$ ), respectively (data not statistically different from the other experimental groups). Rats of the TNBSCysN and CysN groups drank $8.6 \mathrm{ml} /$ day of Cys $/$ water with a daily CysN intake of $8.6 \mathrm{mg}$, and the total CysN dose at the end of the experiment was $189.2 \mathrm{mg}$. No statistically significant differences were found between the drinking volumes of all the study groups.

Compared with the TNBS group, the TNBS-CysN group developed a less severe colitis macroscopically characterized by erythema and small erosions from the right to the left colon, respectively, and microscopically by a decreased inflammation (Table 2). No statistically significant differences were found between the macroscopic and microscopic scores of the CysN and CTR groups.

CysN effect was associated with decreased levels of TG activity and TNF- $\alpha$ (Figures 3 and 4). Moreover, the immunohistochemical evidence of isopeptide bond formation was decreased in CysN rats as demonstration of a reduced activation of TG2 (Figure 5). Rats administered CysN and not receiving the TNBS enema did not express any 

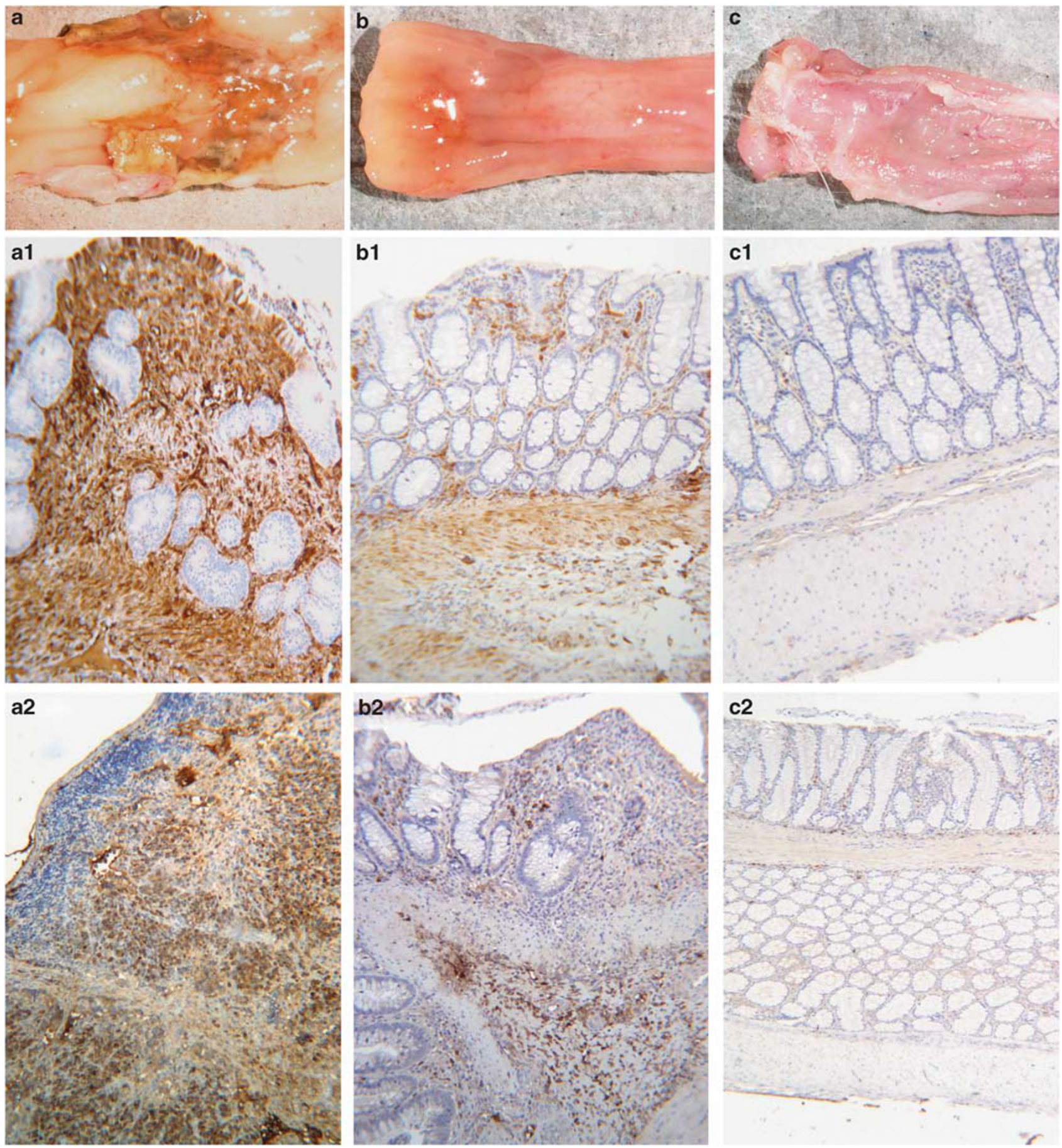

Figure 3 Macroscopic appearance of the colon extracted from rats administered intracolonic 2,4,6-trinitrobenzene sulfonic acid (TNBS) (a), TNBS plus cystamine (CysN) (b) and CTR (c). Immunolocalization of transglutaminase type 2 (TG2) in the colonic mucosa of TNBS (a 1), TNBS plus CysN (b1) and CTR (c1) rats. Immunolocalization (brown staining) of tumor necrosis factor- $\alpha$ (TNF- $\alpha$ ) in the colonic mucosa of TNBS (a2), TNBS plus CysN (b2) and CTR (c2) rats.

damage and presented TG and TNF- $\alpha$ values not different from CTRs. At the same time, IL-6 serological levels were normalized in the rats of the TNBS-CysN group (Figure 6).

At IAR, the TG2 expression on the luminal surface of the colon in the TNBS-CysN rats was increased compared with controls, corresponding to the general area where macroscopically evaluated erosions were found: a more intense response was associated with deeper erosions (Figure 7).

The CysN protective effect was irrelevant in TNBSCysN-24h group that evidenced a severe colitis without 
statistically different scores compared with TNBS-24 h group (macroscopic score median 3, range 3 to 4 , microscopic score 18, 4-19; Figure 2). Also, average mucosal levels of
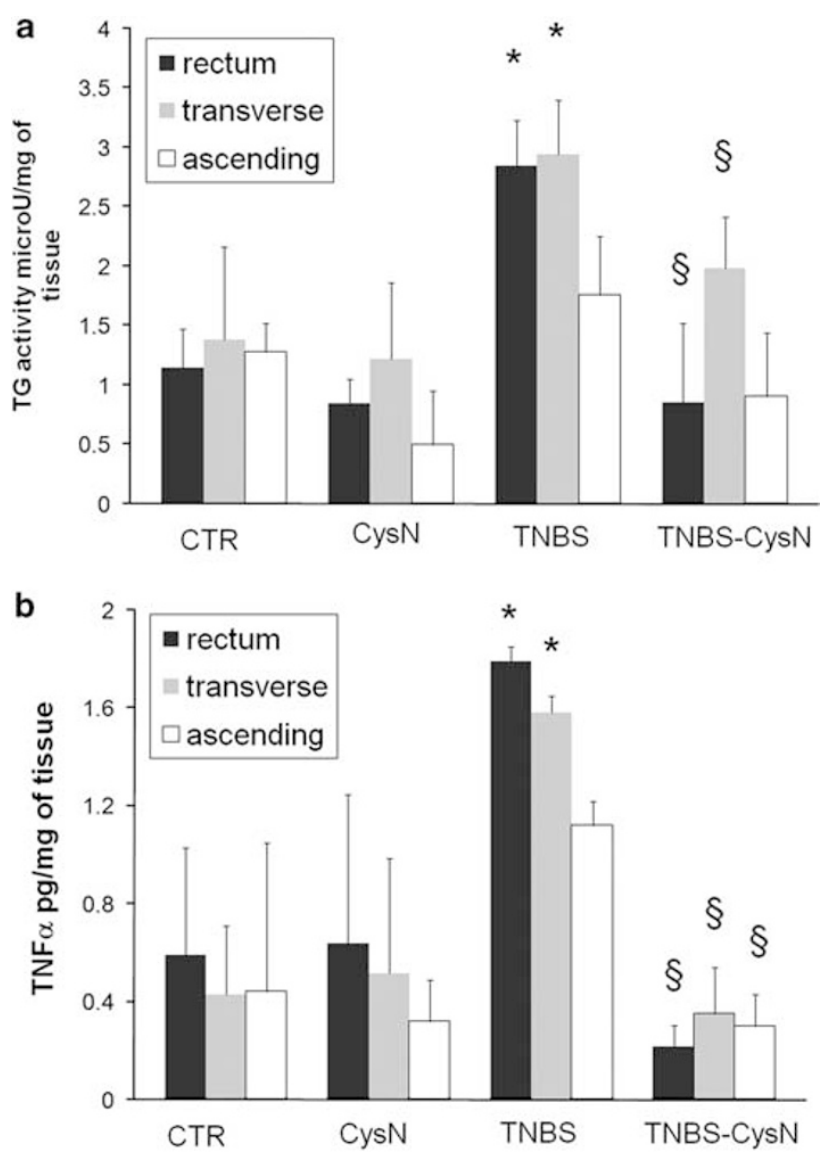

Figure 4 Transglutaminase (TG) activity (a) and tumor necrosis factor- $\alpha$ levels (b) in the colonic mucosa of the different study groups (CTR and CysN, $n=7$; TNBS and TNBS-CysN, $n=8$ ). TNBS, 2,4,6-trinitrobenzene sulfonic acid; CysN, cystamine; CTR, controls. $(\mathbf{a}, \mathbf{b}){ }^{\star} P<0.05$ vs CTR; ${ }^{\$_{P}}<0.05$ vs TNBS.
TNF- $\alpha$, TG activity and serological levels of IL- 6 were not different from those observed in the TNBS- $24 \mathrm{~h}$ group $(2.00 \pm 0.27 \mathrm{pg} / \mathrm{mg}$ of tissue, $3.36 \pm 0.54 \mathrm{microU} / \mathrm{mg}$ of tissue and $6.92 \pm 0.35 \mathrm{pg} / \mathrm{ml}$, respectively).

The TG2 expression in CTR and CysN-administered rats was in accordance with the immunohistochemical analysis of the colonic endoscopic biopsies from the humans studied; in fact, in healthy humans TG2 was decreased or nearly absent compared with IBD patients (data not shown).

\section{DISCUSSION}

For the first time, this study provides data suggesting a possible role of CysN in the treatment of IBD. The antiinflammatory effect of CysN, confirmed by the decreased levels of mucosal TNF- $\alpha$ and serological IL-6, was associated with normalization of TG activity.

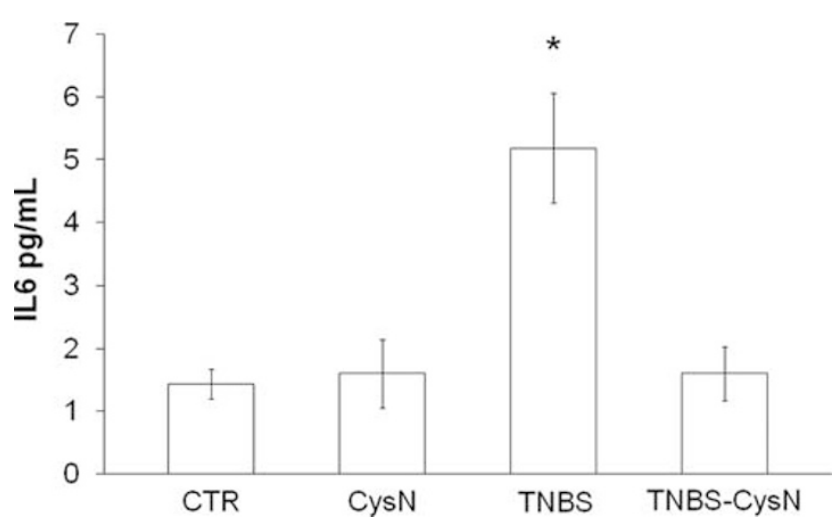

Figure 6 Serological levels of interleukin- 6 in the different study groups (CTR and CysN, $n=7$; TNBS and TNBS-CysN, $n=8) .{ }^{\star} P<0.05$ vs TNBS-CysN, CTR and CysN groups.

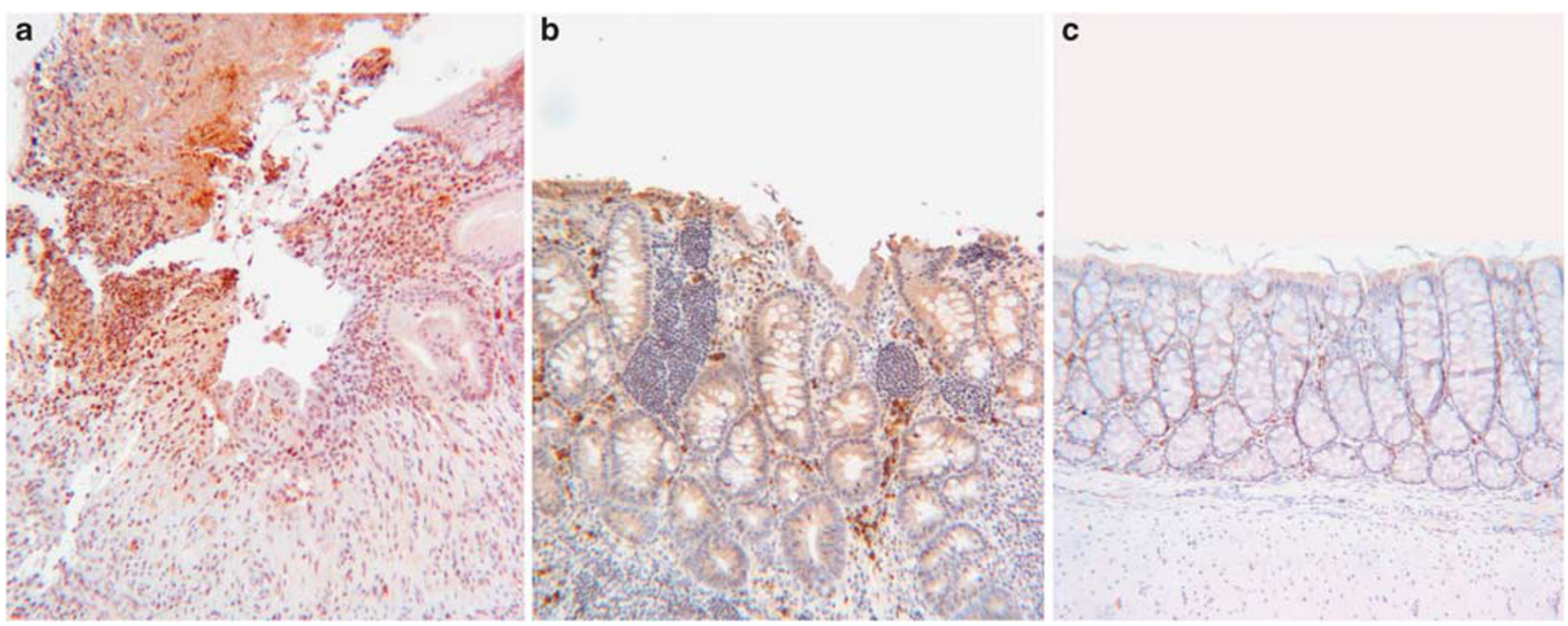

Figure 5 Immunolocalization of isopeptide bonds in the colonic mucosa of TNBS (a), TNBS-CysN (b) and CTR (c) groups. 

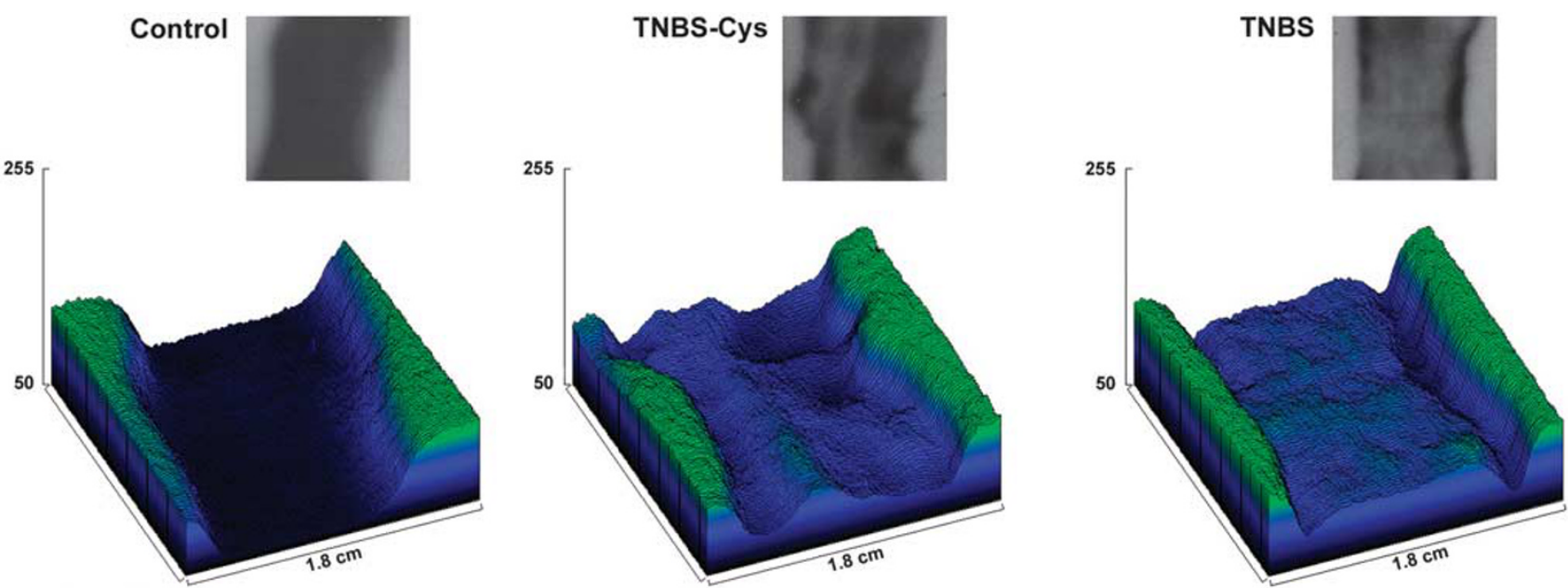

Figure 7 (Top panels) Original autoradiographic images obtained from CTR $(n=7)$, TNBS-CysN $(n=8)$ and TNBS groups $(n=8)$; B\&W images are reported. (Bottom panels) Surface plots converted as 3D images obtained from the original ones showing the transglutaminase type 2 localization and signal intensity. To clarify and evidence the signal, images were recoded using a color scale with two colors in the ramp: blue (assigned to black and no signal) and green (assigned to white and presence of signal).

CysN is a health-promoting compound with negligible side effects at therapeutic doses, acting on different pathways involved in inflammation. ${ }^{28} \mathrm{CysN}$ is able to reduce apoptotic processes by the inhibition of the thiol-dependent protease caspase 3, a pivotal downstream enzyme in the programmed cell death, ${ }^{7}$ and to ameliorate redox balance through the increase of the intracellular glutathione levels with a consequent increase of the reductive power. ${ }^{29}$ However, caspase inhibition has been demonstrated only in vitro, ${ }^{7}$ and increase of glutathione levels could be modest. ${ }^{30,31}$ Besides these effects, CysN is also a relevant inhibitor of the TGs and in particular of TG2, the most-studied enzyme. TG2 is a demidating/transamidating pleiotropic enzyme, deeply involved in inflammatory processes ${ }^{32}$ and central in the pathogenesis of several diseases such as celiac disease and IBD. ${ }^{33,34}$ CysN inhibits TG2 by different mechanisms; in fact, it is a diamine acting not only as a amine substrate competing with natural, endogenous substrates (competitive inhibition) but also as an irreversible inhibitor. This latter effect could be linked to the formation of stable complexes between TG2 active site and Cys $\mathrm{N}$ or by the oxidation of the disulfide bond in the TG2. ${ }^{8,31}$ Moreover, CysN-derived metabolite cystamine $(\beta$-mercaptoethanolamine (MEA)) is a TG2 inhibitor able to compete for transamidation and, theoretically, to limit TG2 expression by reducing the binding of transcription factors to promoters. ${ }^{11}$

In IBD, TG2 and isopeptide bonds are increased in the inflamed mucosa, mainly in the basal membrane and extracellular matrix. ${ }^{17,34}$ Moreover, the increased mucosal TG2 is associated with a decreased activity of the circulating TG (factor XIII, FXIII), which presents an inverse correlation with the disease severity. ${ }^{35,36}$ However, in the literature, data on a possible therapy with FXIII (in order to obtain a colonic increase of the transamidating potential) or with TG2 inhibitors are conflicting. ${ }^{37}$ In fact, in the TNBS rat model of IBD, the intravenous administration of FXIII resulted in effectively reducing the mucosal lesions together with restoration of the serologic TG activity. ${ }^{38}$ From the other hand, in humans the same treatment had no efficacy in the unique controlled available trial. ${ }^{39}$ TG2 inhibition was tested in vivo by Sohn et al, ${ }^{40}$ who demonstrated that TG2-mediated PLA2 modification has a strong antiinflammatory effect. Other data showed a decreased multiorgan injury in TG2 knockout mice affected by a septic shock, mainly through the inhibition of TNF- $\alpha$ pathway, which could also be downregulated by MEA through the DNA binding attenuation of NF- $\kappa \mathrm{B}^{41,42}$

Compared with the results obtained by D'Argenio et $a l^{38}$ after FXIII infusion in the TNBS animal model of IBD, our data suggest that decrease of TG mucosal activity also reduces inflammation. This could be because of the differences between the two enzymes. Although of the same family, FXIII and TG2 have different localizations and sites of action; FXIII is extracellular and its function is the stabilization of clot and the transamidation of ECM, whereas TG2 is inserted in a complex network involving ECM formation (protein crosslinking), inflammation and apoptosis where its actions are exclusive and not substitutable with other members of the TG family. Thus, TG2 inhibition could exert a potent antiinflammatory effect without decreasing the tissue repairing capacity of the mucosa.

In our experiments, after exclusion of possible chemical interactions between TNBS acid and CysN, as demonstrated by short time experiments, TNBS colitis recovered by the CysN oral administration in association with a reduction of proinflammatory factors, TNF- $\alpha$, linked to the mucosal 
injury in IBD and serological IL-6. ${ }^{1,43}$ Although the reduction of TG mucosal activity and TG2 immunohistochemical expression could be secondary to an out-of-target CysN antiinflammatory effect, this seems excluded by isopeptide bond analysis that demonstrated an in situ reduction of TG2 catalyzing activity. Another interesting finding is the TG2 mucosal distribution; in fact, TG2 is present not only in the submucosal compartment, but also on the luminal surface, as shown by the IAR analysis. This is the first demonstration that TG2 is expressed on the luminal colonic surface, in relationship with the macroscopic pictures. Generally, it is unexpressed in CTRs with intact colonic surface (very low signal intensity), whereas it shows a discrete expression in the TNBS-CysN group with isolated erosions (high intensity signal) and a diffuse expression to all the colonic surface in the TNBS group with ulcerated mucosa (medium-high intensity signal). The increased luminal TG2 in ulcerated/ inflamed mucosa and its high expression in the lamina propria and submucosal compartments demonstrate, together with the isopeptide bonds formation, an attempt of healing through a fibrotic mechanism of protein crosslinking, aimed to the restoration of the intestinal barrier integrity as happens in ulcers and scars repair. ${ }^{9}$ On the other hand, this increase could strongly upregulate the TNF- $\alpha$ pathway, leading to enhanced inflammatory processes. ${ }^{44}$

Although different CysN health-promoting effects could be present in our protocol, as suggested in other studies on Huntington's disease, ${ }^{45}$ the results demonstrate that CysN treatment is able to ameliorate the TNBS-induced colitis, reducing the TG activity and isopeptide bond formation in the colonic mucosa. These data are in line with the previously reported beneficial effects of TG inhibition on inflammation and open the windows to clinical trials confirming this therapeutic approach in humans.

\section{DISCLOSURE/CONFLICT OF INTEREST}

The authors declare no conflict of interest.

1. Podolsky DK. Inflammatory bowel disease. N Engl J Med 2002;347: 417-429.

2. Yamamoto-Furusho JK, Podolsky DK. Innate immunity in inflammatory bowel disease. World J Gastroenterol 2007;13:5577-5580.

3. Tsianos EV, Katsanos K. Do we really understand what the immunological disturbances in inflammatory bowel disease mean? World J Gastroenterol 2009;15:521-525.

4. Domenech E. Inflammatory bowel disease: current therapeutic options. Digestion 2006;73(Suppl 1):67-76.

5. Rutgeerts P, Vermeire S, Van Assche G. Biological therapies for inflammatory bowel diseases. Gastroenterology 2009;136:1182-1197.

6. Noble A, Baldassano R, Mamula P. Novel therapeutic options in the inflammatory bowel disease world. Dig Liver Dis 2008:40:22-31.

7. Lesort M, Lee M, Tucholski J, et al. Cystamine inhibits caspase activity. Implications for the treatment of polyglutamine disorders. J Biol Chem 2003;278:3825-3830.

8. Siegel $\mathrm{M}$, Khosla C. Transglutaminase 2 inhibitors and their therapeutic role in disease states. Pharmacol Ther 2007;115:232-245.

9. Griffin $M$, Casadio R, Bergamini CM. Transglutaminases: nature's biological glues. Biochem J 2002;368(Pt 2):377-396.

10. Elli L, Bergamini CM, Bardella MT, et al. Transglutaminases in inflammation and fibrosis of the gastrointestinal tract and the liver. Dig Liver Dis 2009;41:541-550.
11. Jeitner TM, Pinto JT, Krasnikov BF, et al. Transglutaminases and neurodegeneration. J Neurochem 2009;109(Suppl 1):160-166.

12. Van Raamsdonk JM, Pearson J, Bailey CD, et al. Cystamine treatment is neuroprotective in the YAC128 mouse model of Huntington disease. J Neurochem 2005;95:210-220.

13. Fesus L, Piacentini M. Transglutaminase 2: an enigmatic enzyme with diverse functions. Trends Biochem Sci 2002;27:534-539.

14. Nardacci R, Ciccosanti F, Falasca L, et al. Tissue transglutaminase in HCV infection. Cell Death Differ 2003;10(Suppl 1):S79-S80.

15. Amendola $A$, Fesus $L$, Piacentini $M$, et al. 'Tissue' transglutaminase in AIDS. J Immunol Methods 2002;265:145-159.

16. Collighan RJ, Griffin M. Transglutaminase 2 cross-linking of matrix proteins: biological significance and medical applications. Amino Acids 2009;36:659-670.

17. D'Argenio G, Calvani M, Della Valle N, et al. Differential expression of multiple transglutaminases in human colon: impaired keratinocyte transglutaminase expression in ulcerative colitis. Gut 2005;54:496-502.

18. Sjober K, Eriksson S, Tenngart B, et al. Factor XIII and tissue transglutaminase antibodies in coeliac and inflammatory bowel disease. Autoimmunity 2002;35:357-364.

19. Tursi A. Anti-tissue transglutaminase in inflammatory bowel diseases: an activity disease-related phenomenon? Dig Liver Dis 2006;38:711-712.

20. Jurjus AR, Khoury NN, Reimund JM. Animal models of inflammatory bowel disease. J Pharmacol Toxicol Methods 2004;50:81-92.

21. Kankuri E, Vaali K, Korpela R, et al. Effects of a COX-2 preferential agent nimesulide on TNBS-induced acute inflammation in the gut. Inflammation 2001;25:301-310.

22. San-Miguel B, Crespo I, Kretzmann NA, et al. Glutamine prevents fibrosis development in rats with colitis induced by 2,4,6trinitrobenzene sulfonic acid. J Nutr 2010;140:1065-1071.

23. Neurath M, Fuss I, Strober W. TNBS-colitis. Int Rev Immunol 2000;19:51-62.

24. Morris GP, Beck PL, Herridge MS, et al. Hapten-induced model of chronic inflammation and ulceration in the rat colon. Gastroenterology 1989;96:795-803.

25. Gaudio E, Taddei G, Vetuschi A, et al. Dextran sulfate sodium (DSS) colitis in rats: clinical, structural, and ultrastructural aspects. Dig Dis Sci 1999;44:1458-1475.

26. Roncoroni L, Elli L, Dolfini E, et al. Resveratrol inhibits cell growth in a human cholangiocarcinoma cell line. Liver Int 2008;28:1426-1436.

27. Herman JP, Morrison DG. Immunoautoradiographic and in situ hybridization analysis of corticotropin-releasing hormone biosynthesis in the hypothalamic paraventricular nucleus. J Chem Neuroanat 1996;11:49-56.

28. Toohey Jl. Sulfur metabolism in AIDS: cystamine as an anti-HIV agent. AIDS Res Hum Retroviruses 2009;25:1057-1060.

29. Jokay I, Kelemenics K, Gyuris A, et al. S-methylthio-cysteine and cystamine are potent stimulators of thiol production and glutathione synthesis. Life Sci 1998;62:PL27-PL33.

30. Kovarova H, Pulpanova J. Effect of cystamine on rat tissue GSH level and glutathione reductase activity. Strahlentherapie 1979;155: 875-878.

31. Jeitner TM, Delikatny EJ, Ahlqvist J, et al. Mechanism for the inhibition of transglutaminase 2 by cystamine. Biochem Pharmacol 2005;69: 961-970.

32. Beninati S, Bergamini CM, Piacentini M. An overview of the first 50 years of transglutaminase research. Amino Acids 2009;36:591-598.

33. Schuppan D, Dieterich W. A molecular warhead and its target: tissue transglutaminase and Celiac Sprue. Chem Biol 2003;10:199-201.

34. D'Argenio G, Biancone L, Cosenza V, et al. Transglutaminases in Crohn's disease. Gut 1995;37:690-695.

35. D'Argenio $G$, Cosenza V, Riegler $G$, et al. Serum transglutaminase correlates with endoscopic and histopathologic grading in patients with ulcerative colitis. Dig Dis Sci 2001;46:649-657.

36. Hudson M, Wakefield AJ, Hutton RA, et al. Factor XIIIA subunit and Crohn's disease. Gut 1993;34:75-79.

37. Kim SY. Transglutaminase 2 in inflammation. Front Biosci 2006;11: 3026-3035.

38. D'Argenio G, Grossman A, Cosenza V, et al. Recombinant factor XIII improves established experimental colitis in rats. Dig Dis Sci 2000;45:987-997.

39. Lorenz R, Born P, Classen M. [Substitution of factor XIII concentrate in treatment refractory ulcerative colitis. A prospective pilot study]. Med Klin (Munich) 1994;89:534-537. 
40. Sohn J, Kim TI, Yoon $\mathrm{YH}$, et al. Novel transglutaminase inhibitors reverse the inflammation of allergic conjunctivitis. J Clin Invest 2003 111:121-128.

41. Falasca L, Farrace MG, Rinaldi A, et al. Transglutaminase type II is involved in the pathogenesis of endotoxic shock. J Immunol 2008;180:2616-2624.

42. Goldstone SD, Fragonas JC, Jeitner TM, et al. Transcription factors as targets for oxidative signalling during lymphocyte activation. Biochim Biophys Acta 1995;1263:114-122.
43. Mitsuyama $K$, Sata $M$, Rose-John S. Interleukin-6 trans-signaling in inflammatory bowel disease. Cytokine Growth Factor Rev 2006;17: 451-461.

44. Mohan K, Pinto D, Issekutz TB. Identification of tissue transglutaminase as a novel molecule involved in human CD8+ T cell transendothelial migration. J Immunol 2003;171:3179-3186.

45. Bailey CD, Johnson GV. The protective effects of cystamine in the R6/2 Huntington's disease mouse involve mechanisms other than the inhibition of tissue transglutaminase. Neurobiol Aging 2006;27:871-879. 